\section{Fatores de risco para mortalidade materna em área urbana do Nordeste do Brasil}

\author{
Risk factors for maternal mortality in \\ an urban area of Northeast Brazil
}

Régia Maria Batista Leite 1,2 Thália Velho Barreto de Araújo 2 Rivaldo Mendes de Albuquerque 1 Antônio Ricardo Santos de Andrade 3 Paulo José Duarte Neto 3

\footnotetext{
1 Universidade de Pernambuco, Recife, Brasil. 2 Universidade Federal de Pernambuco, Recife, Brasil. 3 Universidade de Federal Rural de Pernambuco, Garanhuns, Brasil.

Correspondência R. M. B. Leite

Universidade de Pernambuco. Rua Rio Negro 466, apto. 201,

Garanhuns, PE 55296-360,

Brasil.

regialeite@yahoo.com.br
}

\begin{abstract}
A case-control study was conducted to investigate risk factors for maternal mortality in Recife, Pernambuco State, Brazil, in 2001-2005. Cases were 75 maternal obstetric deaths in Recife, identified from the Mortality Information System, investigated and analyzed by an expert committee on maternal mortality. Controls, selected from the Information System on Live Births using systematic sampling, were 300 women living in Recife whose last pregnancy occurred during the same period and ended in live births. Increased risk of maternal death was associated with use of the public health system $(O R=4.47 ; 95 \% C I: 1.87$ 10.29), age $\geq 35$ years (OR $=3.06$; 95\%CI: 1.595.92), < 4 years of schooling $(O R=4.95 ; 95 \% C I$ : 2.43-10.08), cesarean section $(O R=3.06$; 95\%CI: 1.77-5.29), and lack of prenatal care or fewer than four prenatal visits (OR = 9.78; 95\%CI: 5.5217.34). The results confirm social inequalities in maternal mortality in Recife and indicate the need to improve healthcare for women during the prenatal period, delivery, and postpartum.
\end{abstract}

Maternal Mortality; Risk Factors; Case-Control Studies

\section{Introdução}

Em muitos países, as mortes relacionadas à gravidez são importantes causas de morte de mulheres em idade reprodutiva 1 . A mortalidade materna é um indicador do acesso à atenção obstétrica de qualidade e das condições de vida das mulheres ${ }^{2}$, registrando-se grandes disparidades entre regiões e países. Em 2000, a Razão de Morte Materna (RMM) foi estimada em seis óbitos por 100 mil nascidos vivos no Canadá, enquanto no Haiti essa Razão foi de 680/100 mil nascidos vivos 2 .

A Organização das Nações Unidas (ONU) propôs, na Declaração para o Desenvolvimento do Milênio, a redução da mortalidade materna em três quartos até 2015, tendo como base o ano de 1990 3. Entretanto, os níveis permanecem elevados em alguns países, com disparidades entre nações 4. No Brasil, em 2003, a RMM corrigida foi de 72,4/100 mil nascidos vivos 5 , superior a países mais pobres e com renda per capita inferior como o Uruguai e Cuba ${ }^{6}$.

No Brasil, as principais causas de óbitos em 2003 foram as obstétricas diretas, sobressaindose as doenças hipertensivas e as síndromes hemorrágicas 5 . No Recife, Pernambuco, no período entre 2001 e 2004, predominaram essas mesmas causas, acrescidas das infecções 7 .

Os fatores determinantes das mortes maternas por causas diretas operam em vários níveis, englobando o menor status social e as condições 
socioeconômicas das mulheres, que definem o acesso à educação, bens e serviços, incluindo serviços de saúde de qualidade ${ }^{2}$. Estudos têm revelado como fatores de risco para a mortalidade materna a idade materna mais elevada 8 , o menor nível de escolaridade 9 , o tipo de ocupação, o número reduzido de consultas de pré-natal 10 , a ausência de companheiro e as condições prévias de saúde 11.

A maioria dos estudos sobre o tema no Brasil teve como propósito estimar a RMM e/ou descrever o perfil das causas. Esta pesquisa teve como objetivo investigar fatores de risco para mortalidade materna obstétrica no Município do Recife.

\section{Método}

Este estudo foi realizado no Recife, capital do Estado de Pernambuco, Nordeste do Brasil. Em 2005, a população do município foi estimada em 1.525.181 habitantes, sendo 35,8\% mulheres em idade fértil 12 .

Realizou-se um estudo caso-controle, tendo como casos todos os óbitos maternos obstétricos ocorridos até um ano após a gestação entre as mulheres residentes no Recife, registrados entre primeiro de janeiro de 2001 e 31 de dezembro de 2005. Foram identificados 76 óbitos maternos, sendo 75 por causa obstétrica e um óbito por causa obstétrica não especificada, que foi excluído do estudo. O óbito materno foi definido como resultante de complicações obstétricas relacionadas à gravidez, parto e puerpério, devido a intervenções, omissões ou tratamento incorreto (causas diretas), ou aquele decorrente de doenças preexistentes ou que se desenvolveram durante a gestação e que foram agravadas pelos efeitos fisiológicos da gravidez (causas indiretas), ocorrido até um ano após o término da gestação 13. No Recife, os óbitos de mulheres em idade fértil, identificados pelo Sistema de Informações sobre Mortalidade (SIM), são investigados pela Secretaria de Saúde pelo uso do método Reproductive Age Mortality Survey (RAMOS) ${ }^{2} \mathrm{e}$ discutidos pelo Comitê Municipal de Estudos da Mortalidade Materna. Até 2003, os óbitos de mulheres entre 10 e 49 anos por causas externas e neoplasias eram excluídos da investigação, e, a partir desse ano, os óbitos por tais causas também passaram a ser investigados.

Os controles corresponderam às mulheres também residentes no Recife e registradas no Sistema de Informações sobre Nascidos Vivos (SINASC) como mãe de nascidos vivos no período de primeiro de janeiro de 2001 e 31 de dezembro de 2005, cuja gravidez não resultou em óbito. Foram identificadas 120.071 mães de nascidos vivos no SINASC, mediante o Programa TabWin 2.2 (Departamento de Informática do SUS. http://portal.saude.gov.br/portal/se/ datasus/area.cfm?id_area=732). Foram excluídas as mulheres identificadas como óbito materno e aquelas cujo registro não dispunha de informação para todas as variáveis estudadas. As variáveis utilizadas na identificação e exclusão dos casos de morte materna foram o nome e a idade da mulher, a data e o local do parto. Dessa forma, restaram 110.538 mulheres no banco de dados, sendo obtidos 300 controles, na razão de 4:1, selecionados de forma sistemática. O intervalo de seleção foi igual a 368 (número total de mulheres dividido pelo número de controles), sendo o primeiro registro escolhido aleatoriamente entre os registros de número 1 a 368 no banco de dados do SINASC. A cada intervalo foi retirada uma unidade amostral, até que o número desejado de controles fosse obtido. Na Figura 1, são apresentados os procedimentos utilizados na construção do banco de dados. O SIM e o SINASC no Recife possuem elevada cobertura e qualidade 14.

As variáveis independentes, selecionadas com base em relatos da literatura e da disponibilidade nos bancos de dados, foram: idade (em anos), escolaridade (anos completos de estudo), inserção produtiva (engajada ou não em atividade produtiva), parição (nulíparas - sem filho anterior-e multíparas - 1 ou mais filhos anteriores, nascidos vivos ou mortos), tipo de parto (vaginal ou cesariano), realização e número de consultas de pré-natal e tipo de provedor do estabelecimento de saúde (SUS/não SUS). A rede SUS inclui as maternidades próprias ou conveniadas, as instituições universitárias e as filantrópicas.

Inicialmente, foi efetuada a distribuição de frequências absoluta e relativa dos óbitos por causas. Realizou-se uma análise univariada dos dados por meio do programa Epi Info 6.0 (Centers for Disease Control and Prevention, Atlanta, Estados Unidos). Foram estimadas odds ratios (OR) como medida de magnitude da associação entre a chance de morte materna e as covariáveis de exposição estudadas com intervalos de 95\% de confiança (IC95\%). A OR foi utilizada como aproximação do risco relativo. Para a avaliação de significância estatística das associações encontradas, utilizou-se o teste de Mantel-Haenszel, com nível de 5\% de significância $(\mathrm{p}<0,05)$.

A análise de regressão logística não condicional foi realizada para obter OR ajustadas para as variáveis que apresentaram associação estatisticamente significativa na etapa anterior da análise. O critério estatístico adotado para a inclusão ou exclusão definitiva de variáveis dos modelos 
Estruturação final do banco de dados.

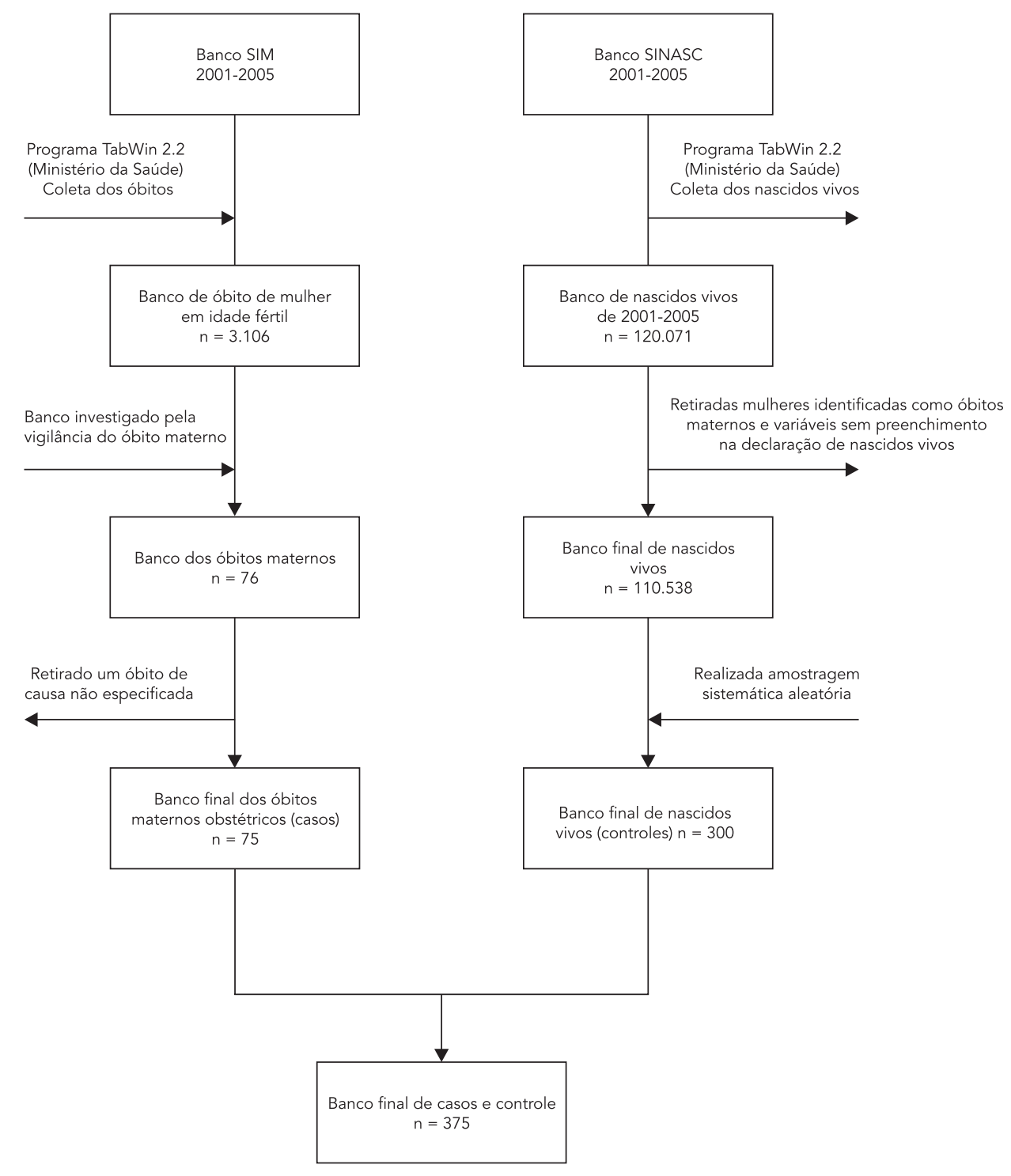

SIM: Sistema de Informações sobre Mortalidade; SINASC: Sistema de Informações sobre Nascidos Vivos.

multivariados foi a obtenção de valores de $\mathrm{p}<$ 0,05 para o teste de Wald.

A pesquisa foi aprovada pelo Comitê de Ética em Pesquisa da Universidade Federal de Pernambuco (protocolo no. 093/2005). Foi preservada a confidencialidade dos dados e o anonimato das mulheres, das instituições de saúde, dos familiares das mulheres e de todas as outras pessoas que contribuíram com alguma informação durante o processo de investigação do óbito.

\section{Resultados}

Todas as mulheres que foram a óbito por causas maternas tinham idade entre 16 e 43 anos (média de 28,7 anos e desvio padrão - DP $=7,5$ ), em sua maioria vivia em união, era de cor parda $(69,7 \%)$, não exercia atividade remunerada e possuía menor escolaridade. Aproximadamente $17 \%$ não fizeram pré-natal, e $26,2 \%$ realizaram seis ou mais consultas. 
A distribuição das causas dos óbitos obstétricos diretos e indiretos é apresentada na Tabela 1 . As causas obstétricas diretas responderam por $54,7 \%$ das mortes maternas e as indiretas, por $45,3 \%$. Dentre as causas obstétricas diretas, predominaram as doenças hipertensivas, as infecções, as hemorragias, as cardiomiopatias pósparto e os abortamentos, ao passo que entre as causas obstétricas indiretas prevaleceram a AIDS, as infecções e as cardiopatias preexistentes.

Na Tabela 2, são apresentados os resultados da análise univariada. A inserção produtiva, a situação conjugal e a parição não se mostraram associadas à morte materna, no nível de significância de 5\%, não tendo sido incluídas na análise multivariada.

$\mathrm{Na}$ análise multivariada, as variáveis tipo de provedor do serviço de saúde onde ocorreu o óbito, idade da mulher ( $\geq 35$ anos), tipo de parto (cesárea) e número de consultas de pré-natal mostraram-se associadas ao óbito materno, conforme apresentado na Tabela 3 . Ainda que a chance de óbito tenha sido mais de duas vezes maior ( $\mathrm{OR}=2,19)$ para as mulheres com menor escolaridade ( $<4$ anos de estudo), esta associação não alcançou significância estatística de $5 \%$. Nessa última etapa da análise, quando da introdução da variável "número de consultas de prénatal", a variável "realização do pré-natal” foi excluída do modelo, possivelmente, por expressar o mesmo fator de risco.

\section{Discussão}

Este estudo tem a vantagem de explorar fatores de risco para morte materna em uma área geograficamente bem delimitada, pois se trata de caso-controle de base populacional. Por outro lado, são raros na produção científica brasileira 15 os estudos de morte materna que mensuram o risco de morte associados às variáveis estudadas. Ademais, foi realizado em uma capital onde a proporção de partos não hospitalares é bastante reduzida (Departamento de Informática do SUS. Informações de Saúde. http:// tabnet.

\begin{tabular}{|c|c|c|}
\hline Causa dos óbitos (CID-10) & n & $\%$ \\
\hline Obstétricas diretas & 41 & 54,7 \\
\hline Transtornos hipertensivos (O12-O16) & 17 & 22,7 \\
\hline Hemorragias (O46.9, 047.9, 072.0-O72.3) & 6 & 8,0 \\
\hline Infecções (085, 086.0-086.8) & 5 & 6,7 \\
\hline Miocardiopatia periparto (О90.3) & 4 & 5,3 \\
\hline Aborto (O00-O08) & 4 & 5,3 \\
\hline Complicações anestésicas (O89.0-O89.9, O74.2, О29.1) & 1 & 1,3 \\
\hline Psicose puerperal (F53) & 1 & 1,3 \\
\hline Neoplasia trofoblástica gestacional (D39.2) & 1 & 1,3 \\
\hline Doenças do aparelho respiratório (O99.5) & 1 & 1,3 \\
\hline Tromboembolismo (O88.2) & 1 & 1,3 \\
\hline Obstétricas indiretas & 34 & 45,3 \\
\hline AIDS (B20-B24) & 7 & 9,4 \\
\hline Infecções (exceto AIDS e Tuberculose) (O98.1-O98.9) & 7 & 9,4 \\
\hline Cardiopatias preexistentes (I00-109) & 7 & 9,4 \\
\hline Doenças do sangue (099.0-099.1) & 3 & 4,0 \\
\hline Hipertensão arterial sistêmica crônica (O10-011) & 3 & 4,0 \\
\hline Tuberculose (О98.0) & 2 & 2,7 \\
\hline Doenças do aparelho digestivo (О99.6) & 2 & 2,7 \\
\hline Doenças do aparelho respiratório (O99.5) & 1 & 1,3 \\
\hline Neurofibromatose (O99.8) & 1 & 1,3 \\
\hline Diabetes preexistente (О९9.2) & 1 & 1,3 \\
\hline
\end{tabular}

CID-10: Classificação Internacional de Doenças - 10ạ Revisão. 
Tabela 2

Análise univariada dos fatores de risco para mortalidade materna por causas obstétricas de mulheres residentes no Recife,

Pernambuco, Brasil, 2001-2005.

\begin{tabular}{|c|c|c|c|c|c|}
\hline Fatores de risco & $\begin{array}{l}\text { Casos } \\
\text { n (\%) }\end{array}$ & $\begin{array}{c}\text { Controles } \\
\text { n (\%) }\end{array}$ & $\begin{array}{l}\text { Total } \\
\text { n (\%) }\end{array}$ & OR (IC95\%) & Valor de $p$ \\
\hline \multicolumn{6}{|l|}{ Inserção produtiva } \\
\hline Sim & $64(85,3)$ & $223(74,3)$ & $287(76,5)$ & $2,00(1,00-4,00)$ & 0,063 \\
\hline Não & $11(14,7)$ & $77(25,7)$ & $88(23,5)$ & & \\
\hline \multicolumn{6}{|l|}{ Idade (anos) } \\
\hline$<35$ & $57(76,0)$ & $272(90,7)$ & $329(87,7)$ & $3,06(1,59-5,92)$ & 0,001 \\
\hline$\geq 35$ & $18(24,0)$ & $28(9,3)$ & $46(12,3)$ & & \\
\hline \multicolumn{6}{|l|}{ Escolaridade (anos) } \\
\hline$<4$ & $18(24,0)$ & $18(6,0)$ & $36(9,6)$ & $4,95(2,43-10,09)$ & 0,001 \\
\hline$\geq 4$ & $57(76,0)$ & $282(94,0)$ & $339(90,4)$ & & \\
\hline \multicolumn{6}{|l|}{ Situação conjugal } \\
\hline Solteira/Divorciada/Viúva & $33(44,0)$ & $129(43,0)$ & $162(43,2)$ & $1,04(0,625-1,734)$ & 0,979 \\
\hline Casada/Unida & $42(66,0)$ & $171(57,0)$ & $213(56,8)$ & & \\
\hline \multicolumn{6}{|l|}{ Realização do pré-natal } \\
\hline Não & $13(17,3)$ & $9(3,0)$ & $22(5,9)$ & $6,78(2,78-16,56)$ & 0,000 \\
\hline Sim & $62(82,7)$ & $291(97,0)$ & $353(94,1)$ & & \\
\hline \multicolumn{6}{|l|}{ Número de consultas } \\
\hline $0-3$ & $44(58,7)$ & $38(12,7)$ & $82(21,9)$ & $9,78(5,52-17,34)$ & 0,000 \\
\hline$\geq 4$ & $31(41,3)$ & $262(87,3)$ & $293(78,1)$ & & \\
\hline \multicolumn{6}{|l|}{ Parição } \\
\hline Nulípara & $30(40,0)$ & $133(44,3)$ & $163(43,4)$ & $1,19(0,71-1,99)$ & 0,585 \\
\hline Multípara & $45(60,0)$ & $167(55,7)$ & $212(53,6)$ & & \\
\hline \multicolumn{6}{|l|}{ Tipo de parto } \\
\hline Parto vaginal & $22(29,3)$ & $168(56,0)$ & $190(50,67)$ & $3,06(1,77-5,29)$ & 0,000 \\
\hline Parto cesáreo & $53(70,7)$ & $132(44,0)$ & $185(49,33)$ & & \\
\hline \multicolumn{6}{|l|}{ Tipo de provedor } \\
\hline SUS & $69(92,0)$ & $216(72,0)$ & $285(76,0)$ & $4,47(1,87-10,69)$ & 0,001 \\
\hline Não SUS & $6(8,0)$ & $84(28,0)$ & $90(24,0)$ & & \\
\hline
\end{tabular}

IC95\%: intervalo de 95\% de confiança; OR: odds ratio.

Tabela 3

Modelo de regressão múltipla para o risco da mortalidade materna por causas obstétricas. Recife, Pernambuco, Brasil, 2001-2005

\begin{tabular}{lcccccc}
\hline Variáveis & OR bruta & IC95\% & Valor de p & OR $_{\text {ajustada }}$ & IC95\% & Valor de p \\
\hline Tipo de provedor (SUS/não SUS) & 4,47 & $1,87-10,29$ & 0,001 & 6,73 & $2,41-18,79$ & 0,000 \\
Idade $(\geq 35 /<35$ anos) & 3,06 & $1,59-5,92$ & 0,001 & 3,33 & $1,45-7,68$ & 0,005 \\
Escolaridade (<4/ $\geq 4)$ & 4,95 & $2,43-10,08$ & 0,001 & 2,19 & $0,84-5,73$ & 0,108 \\
Tipo de parto (cesáreo/normal) & 3,06 & $1,77-5,29$ & 0,000 & 7,08 & $3,54-14,17$ & 0,000 \\
Número de consultas (<3/ $\geq 4)$ & 9,78 & $5,52-17,34$ & 0,000 & 8,84 & $4,45-17,56$ & 0,000 \\
\hline
\end{tabular}

IC95\%: intervalo de 95\% de confiança; OR: odds ratio. 
datasus.gov.br/cgi/tabcgi.exe?sinasc/cnv/nvpe. def, acessado em Mai/2011), pois possui sistema de vigilância do óbito materno e boa cobertura do SINASC 14.

Por outro lado, o estudo tem como limitação o fato de se tratar de análise realizada com dados secundários, o que restringiu a exploração dos fatores de risco ao elenco de variáveis disponível nas bases de dados utilizadas. Por isso, não foi considerado o efeito de algumas variáveis importantes, como raça, complicações ocorridas na gravidez e comorbidades preexistentes.

A redução da mortalidade materna depende do efetivo monitoramento dos casos, fato que nem sempre é possível por conta da subinformação, que impede o reconhecimento do óbito como materno, e ao sub-registro ${ }^{16}$. Em período recente, o sistema de informação do Recife tem permitido dimensionar de forma clara a magnitude da mortalidade materna. A identificação de grupos de maior risco pode contribuir para a prevenção desses óbitos.

As cinco principais causas de morte materna obstétrica direta no Recife não se modificaram na última década, considerando-se que em pesquisa anterior, realizada no período de 1994-2000, encontrou-se perfil idêntico 17. Situação semelhante também foi evidenciada para o Estado de Pernambuco, embora as mortes por complicações do aborto venham ampliando a sua participação entre as causas de óbito materno ${ }^{18}$. Dentre as causas obstétricas indiretas, o estudo mostra o avanço da AIDS, que já ocupa a primeira posição neste grupo, juntamente com outras infecções e as doenças cardiovasculares preexistentes.

Em relação aos fatores demográficos e socioeconômicos, as mulheres de 20-34 anos de idade predominaram entre os casos, o que seria esperado, considerando-se que nessa faixa etária ocorre o maior número de gravidezes 19 . Porém, o risco de morte elevou-se com a idade, acentuando-se bastante a partir dos 35 anos ou mais. Estudos conduzidos no México, no Chile e em países da África também mostraram maior risco de morte materna em mulheres com idades mais avançadas 20,21,22. O risco de morte também foi maior para as mulheres com menos de quatro anos de estudo, semelhantemente a outras capitais pesquisadas no Brasil 16.

Em sociedades com grandes iniquidades no acesso à educação formal, como no Brasil, o nível de escolaridade se constitui um indicador do nível socioeconômico 16,23. A conjunção de menor escolaridade e condição de vida desfavorável pode dificultar o acesso à informação e aos cuidados de saúde necessários ao desenvolvimento de uma gravidez saudável, além de restringir o reconhecimento de direitos de cidadania. A mor- talidade materna consiste em um dos indicadores de saúde com maior desigualdade entre países, regiões e segmentos sociais, de acordo com o nível de desenvolvimento social e acumulação de riqueza ${ }^{3}$.

A situação conjugal não se mostrou associada ao risco de morte materna no presente estudo, divergindo do que foi verificado em pesquisa semelhante realizada no México 24 . Entretanto, não é possível excluir a possibilidade de que tal ausência de associação tenha resultado do fato de que a informação obtida mescla a situação conjugal com o estado civil, por se tratar de dados secundários. A presença do companheiro tem sido interpretada como a possibilidade de maior suporte emocional e apoio material no período da gravidez 25 .

As mulheres que declararam ter uma inserção produtiva apresentaram maior chance de morte relacionada à gravidez, ainda que as mulheres sem atividade remunerada predominem entre os casos de óbito. Possuir uma atividade produtiva pode não representar uma situação econômica mais favorável, pois a participação no mercado de trabalho representa, para muitas mulheres, uma estratégia necessária à complementação da renda familiar ou, ainda, à manutenção dela e de sua família. Para essas mulheres, faz-se necessário conciliar o trabalho remunerado com a realização de atividades domésticas e o cuidado dos filhos. Além disso, e talvez por isso, as mulheres com atividade remunerada possam procurar menos os serviços de saúde, ainda quando da ocorrência de problema de saúde 26 .

A não realização de pré-natal e o número insuficiente de consultas estão fortemente associados ao risco de morte materna na população estudada. É importante destacar que apenas 26,2\% das mulheres realizaram seis consultas ou mais, número mínimo proposto pelo Programa de Humanização no Pré-natal e Nascimento (PHPN) do Ministério da Saúde 27. Sabe-se que a redução da morbimortalidade materna depende, em grande parte, da realização do pré-natal 8,10,15,20 e da qualidade da atenção recebida na gravidez, parto e puerpério ${ }^{4}$. Em estudo caso-controle sobre os determinantes da morte materna, realizado no Município de Fortaleza, a dificuldade de acesso aos serviços de saúde foi relatada por $19,6 \%$ das mulheres do grupo controle, enquanto para os casos, este percentual foi de $41,1 \% 15$.

No Recife, em 2008, a cobertura de pré-natal foi estimada em $98,1 \%$, embora tenha sido registrado menor acesso para as mulheres com menor nível de escolaridade (DATASUS. http://tabnet. datasus.gov.br/cgi/tabcgi.exe?sinasc/cnv/nvpe. def, acessado em Mai/2011). Um estudo anterior realizado no Recife, em 2004, também verificou 
elevada cobertura de pré-natal e apontou a persistência de barreiras ao cumprimento das normas da atenção pré-natal, especialmente na realização dos exames de rotina e, paradoxalmente, no cumprimento dos procedimentos clínicoobstétricos preconizados 28 . O fato de as causas obstétricas diretas responderem por $54,7 \%$ do total dos óbitos indica que a assistência pré-natal e ao parto ainda requer melhoria de qualidade.

As multíparas não apresentaram risco de morte maior do que as nulíparas. Possivelmente isso se deva à redução do número de filhos por mulher em idade fértil observado nas últimas décadas no Brasil 19.

A proporção de cesarianas foi mais elevada entre os casos do que entre os controles, após o ajuste pelas demais covariáveis, com risco mais elevado para as mulheres que tiveram parto cesariano. Ainda que a presença de morbidade obstétrica e a indicação do procedimento não tenham sido consideradas na análise, os achados deste estudo sugerem que a realização de parto cirúrgico pode contribuir para elevar o risco de morte materna. Outros estudos que investigaram a associação do tipo de parto com o risco de morte materna evidenciaram igual associação 18,20, mesmo quando da ausência de morbidade obstétrica 15,18,20. Na Região Nordeste do Brasil, a proporção de partos cirúrgicos foi estimada em $32 \%$ no ano de 2006 19, tendo sido, portanto, muito superior ao limite de $15 \%$ recomendado pela OMS 29 .

As mulheres usuárias do SUS também apresentaram um risco de morte mais elevado do que as usuárias de serviços privados. Todavia, este achado deve ser interpretado com cautela, pois mais de $85 \%$ dos partos das mulheres do Nordeste do Brasil são realizados em unidades do SUS 19 e, certamente, as mulheres procedentes dos segmentos sociais menos favorecidos, que não realizam pré-natal adequado e que por vezes chegam aos serviços de saúde em estado grave, estão mais presentes entre essa clientela 7 .

Em síntese, os resultados deste estudo reafirmam a perpetuação das iniquidades sociais na determinação da mortalidade materna no Recife. Além disso, apontam para a existência de um paradoxo, por se tratar de área urbana onde a captação para o pré-natal é elevada e a quase totalidade dos partos e dos óbitos maternos ocorrem no âmbito dos serviços de saúde. Ainda que tenham sido evidenciados alguns dos fatores de risco para mortalidade materna, há questões muito complexas na construção desses óbitos que ainda precisam ser mais bem conhecidas, como aspectos referentes à qualidade da atenção recebida na gravidez, no parto e no puerpério.

\section{Resumo}

Este estudo de caso-controle analisou fatores de risco para mortalidade materna no Recife, Pernambuco, Brasil, no período de 2001-2005. Os casos foram 75 óbitos maternos obstétricos, identificados no Sistema de Informações sobre Mortalidade, investigados e analisados pelo Comitê de Mortalidade Materna. Os controles, selecionados no Sistema de Informações sobre Nascidos Vivos, pela amostra sistemática, foram 300 mulheres residentes no Recife cuja última gravidez ocorreu no mesmo período e não resultou em óbito. $O$ risco de morte foi mais elevado para as usuárias do SUS (OR = 4,47; IC95\%: 1,87-10,29), com idade $\geq 35$ anos $(O R=3,06$; IC95\%: 1,59-5,92), < 4 anos de estudo $(O R=4,95 ;$ IC95\%: 2,43-10,08), que tiveram parto cesáreo (OR =3,06; IC95\%: 1,77-5,29) e para aquelas que não realizaram pré-natal ou tiveram menos de 4 consultas (OR = 9,78; IC95\%: 5,52-17,34). Os resultados reafirmam as desigualdades sociais na determinação de mortalidade materna no Recife. Além disso, indicam a necessidade de aprimorar a assistência à saúde da gestante durante o pré-natal, parto e puerpério.

Mortalidade Materna; Fatores de Risco; Estudos de Casos e Controles 


\section{Colaboradores}

R. M. B. Leite, T. V. B. Araújo e R. M. Albuquerque participaram na concepção e projeto ou análise e interpretação dos dados, na redação do artigo ou revisão crítica relevante do conteúdo intelectual e na aprovação final da versão a ser publicada. A. R. S. Andrade e P. J. Duarte Neto contribuíram com a concepção e projeto ou com a análise e interpretação dos dados, além de aprovarem a versão final a ser publicada.

\section{Referências}

1. Family Care International/Safe Motherhood InterAgency Group. Safe motherhood fact sheets: maternal mortality. http://www.safemotherhood.org/ facts_and_figures/maternal_mortality.html (acessado em Jul/2007).

2. World Health Organization/United Nations Children's Fund/United Nations Population Fund/ World Bank. Maternal mortality in 2000: estimates developed by WHO, UNICEF, UNFPA and The World Bank. Geneva: World Health Organization; 2005.

3. United Nations Millennium Declaration. Resolution A/RES/55/2. New York: United Nations; 2000.

4. World Health Organization/United Nations Children's Fund/United Nations Population Fund/ World Bank. Reduction of maternal mortality. http://www.who.int/reproductivehealth/publica tions/reduction_of_maternal_mortality (acessado em Jul/2010).

\section{Agradecimentos}

Ao corpo técnico da Gerência Operacional de Informação sobre Mortalidade e Natalidade da Diretoria de Vigilância à Saúde da Secretaria de Saúde do Recife, que disponibilizou os dados analisados neste estudo.
5. Ministério da Saúde. Manual dos comitês de mortalidade materna. http://portal.saude.gov.br/por tal/comitês_mortalidade_materna_M.pdf (acessado em Jan/2009).

6. Wong LR, Perpétuo IHO. La transición de la salud sexual y reproductiva en América Latina. 15 años después de El Cairo - 1994. Santiago de Chile: División de Población, Centro Latinoamericano y Caribeño de Demografía; 2011.

7. Leite RMB. Mortalidade materna no Recife: análise das trajetórias das mulheres [Dissertação de Mestrado]. Recife: Universidade Federal de Pernambuco; 2006

8. Mbonye AK. Risk factors associated with maternal deaths in health units in Uganda. Afr J Reprod Health 2001; 5:47-53.

9. Magadi M, Diamond I, Madise N. Analysis of factors associated with maternal mortality in Kenyan hospitals. J Biosoc Sci 2001; 33:375-89. 
10. Taguchi N, Kawabata M, Maekawa M, Maruo T, Aditiawarman, Dewata L. Influence of socio-economic background and antenatal care programs on maternal mortality in Surabaya, Indonesia. Trop Med Int Health 2003; 8:847-52.

11. Garenne M, Mbaye K, Bah MD, Correa P. Risk factors for maternal mortality: a case-control study in Dakar hospitals (Senegal). Afr J Reprod Health 1997; 1:14-24.

12. Instituto Brasileiro de Geografia e Estatística. Censo demográfico 2000. Características gerais da população e instrução. Rio de Janeiro: Instituto Brasileiro de Geografia e Estatística; 2002.

13. Organização Mundial da Saúde. Classificação estatística internacional de doenças e problemas relacionados à saúde. Manual de instrução. 10a revisão. v. 2. São Paulo: Edusp; 2008.

14. Andrade CLT, Szwarcwald CL. Desigualdades sócio-espaciais da adequação das informações de nascimentos e óbitos do Ministério da Saúde, Brasil, 2000-2002. Cad Saúde Pública 2007; 23:1207-16.

15. Almeida NMG. Mortalidade materna em Fortaleza - CE, 1996 a 1999: estudo dos fatores de risco [Tese de Doutorado]. São Paulo: Faculdade de Saúde Pública, Universidade de São Paulo; 2002.

16. Laurenti R, Mello-Jorge MHP, Gotlieb SLD. A mortalidade materna nas capitais brasileiras: algumas características e estimativa de um fator de ajuste. Rev Bras Epidemiol 2004; 7:449-60.

17. Costa AAR, Ribas MSS, Amorim MMR, Santos LC. Mortalidade materna na cidade do Recife. Rev Bras Ginecol Obstet 2002; 24:455-62.

18. Valongueiro SA. Maternal mortality in Pernambuco, Brazil: what has changed in ten years? Reprod Health Matters 2007; 30:134-44.

19. Departamento de Ciência e Tecnologia, Secretaria de Ciência, Tecnologia e Insumos Estratégicos, Ministério da Saúde. Pesquisa nacional de demografia e saúde da criança e da mulher. http://bvsms. saude.gov.br/bvs/publicacoes/pnds_crianca_mu lher.pdf (acessado em Jan/2009).
20. Ferrer-Arreola L, Basavilvazo-Rodríguez MA, Lemus-Rocha R, Toca-Porraz L, Hernández-Valencia M. Analysis of trends in maternal mortality during a 10-year follow-up in an urban region. Ginecol Obstet Mex 2005; 73:477-83.

21. Donoso ES, Villarroel LDP. Edad materna avanzada y riesgo reproductivo. Rev Méd Chile 2003; 131:55-9.

22. Evjen-Olsen B, Hinderaker SG, Lie RT, Bergsj P, Gasheka P, Kvåle G. Risk factors for maternal death in the highlands of rural northern Tanzania: a casecontrol study. BMC Public Health 2008; 8:52-6.

23. Hogan MC, Foreman KJ, Naghavi M, Ahn SY, Wang M, Makela SM, et al. Maternal mortality for 181 countries, 1980-2008: a systematic analysis of progress towards Millennium Development Goal 5. Lancet 2010; 375:1609-23.

24. Romero-Gutiérrez G, Espitia-Vera A, Ponce de León AL, Huerta-Vargas LF. Risk factors of maternal death in Mexico. Birth 2007; 34:21-5.

25. Área Técnica de Saúde da Mulher, Secretaria de Políticas de Saúde, Ministério da Saúde. Gestação de alto risco. 4a Ed. Brasília: Ministério da Saúde; 2000.

26. Gomes KRO, Tanaka ACd'A. Morbidade referida e uso dos serviços de saúde por mulheres trabalhadoras, município de São Paulo. Rev Saúde Pública. 2003; 37:75-82.

27. Serruya SJ, Cecatti JG, Lago TG. O Programa de Humanização no Pré-natal e Nascimento do Ministério da Saúde no Brasil: resultados iniciais. Cad Saúde Pública 2004; 20:1281-9.

28. Carvalho VCP, Araújo TVB. Adequação da assistência pré-natal em gestantes atendidas em dois hospitais de referência para gravidez de alto risco do Sistema Único de Saúde, na cidade do Recife, Estado de Pernambuco. Rev Bras Saúde Matern Infant 2007; 7:309-17.

29. Appropriate technology for birth. Lancet 1985; 2:436-7.

Recebido em 24/Fev/2011

Versão final apresentada em 31/Mar/2011 Aprovado em 27/Jun/2011 